\title{
Antibiotics Medication Prescribed in the Management of Respiratory Tract Infection of Tertiary Care Teaching Hospital
}

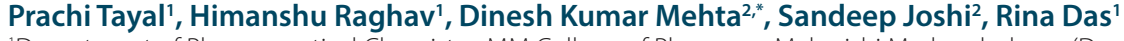 \\ 'Department of Pharmaceutical Chemistry, MM College of Pharmacy, Maharishi Markandeshwar (Deemed to be University), Mullana, Ambala, Haryana, INDIA. \\ 2Department of Medicine, Maharishi Markandeshwar Institute of Medical Sciences and Research, Maharishi Markandeshwar (Deemed to be University), Mullana, \\ Ambala, Haryana, INDIA.
}

\begin{abstract}
Background: Respiratory tract infections (RTIs) encompass a tremendous breadth of clinical disease. Our present study aims to accessing the drug utilization pattern of antibiotic usage in medicine and respiratory unit of tertiary care hospital which helps in accessing rationality and monitoring the pattern of drug prescriptions, drug efficacy and other parameters which access the patient safety. Methods: A prospective observational study was conducted for a period of six months from October 2019 to April 2020 to analyse the drug utilization pattern of the patient who was diagnosed with respiratory tract infections. Results: 111 patients were included as per inclusion and exclusion criteria, out of which $75(67.6 \%)$ were male and $36(32.4 \%)$ were female. The mean $( \pm S D)$ age of the population has been found to be 49 (12.05) years. The majority of patients had hypertension $13(11.71 \%)$ as co-morbidity followed by acute exacerbation 12 (10.81\%). Among 111 cases, 21 (18.9\%) cases were diagnosed with upper respiratory tract infection (URTI) in which $09(42.85 \%)$ patients had Otitis media, 01 (4.76\%) had Supraglottic, 10 (47.61\%) had common cold and 01 $(4.76 \%)$ had Adeno-tonsillitis. In study population 90 (81.1\%) cases were di-
\end{abstract}

agnosed with lower respiratory tract infection (LRTI) in which $49(54.44 \%)$ were COPD, 12 (13.33\%) were B. Asthma, 16 (17.77\%) were Pneumonia. The most commonly prescribed antibiotic was ceftriaxone + sulbactam $73(65.8 \%)$ and azithromycin $72(64.9 \%)$. Conclusion: In a nutshell, it is evident those overuses of antibiotics that were prescribed in the patients of URTI and is a matter of concern. The drug utilization studies are an important tool to sensitize and increases awareness among physicians, which ultimately improves rational prescribing and patient care.

Key words: Antibiotic Drug utilization, Haryana, LRTIs, Rational Use, Resistance, URTIs.

\section{Correspondence}

Prof. Dinesh Kumar Mehta,

MM College of Pharmacy, Maharishi Markandeshwar (Deemed to be University), Mullana, Ambala-133207, Haryana, INDIA.

Email id: dkmehta17@rediffmail.com

DOI: 10.5530/jyp.2021.13.53

\section{INTRODUCTION}

Globally more than 1.5 million deaths occurs annually from respiratory tract infections that are attributable to the environment, including at least $42 \%$ of lower respiratory tract infections and $24 \%$ of upper respiratory tract infections in developing countries. Respiratory tract infections (RTIs) encompass a tremendous breadth of clinical disease which can invasive anywhere in the respiratory tract (i.e. nose, throat and lungs) and is one of the leading cause of morbidity and mortality. ${ }^{1,2}$ The economic and social impact of these infections is significant and constitutes an important challenge for public health, due to high costs concerning treatment, hospitalizations, and loss of working days by patient, caregivers and AMR (anti-microbial resistance) which is the ability of pathogenic bacteria to withstand the actions of antibiotic drugs that arises due to increasing consumption of antibiotics at individual, community, country and regional levels. ${ }^{3-6}$ Increased community consumption of antibiotics is highly interrelated with emerging antibioticresistant infections. Therefore, the conditions where more prescribing of broad-spectrum antibiotics, specifically extended-spectrum cephalosporins and macrolides, rates of multidrug-resistant pneumococcal disease are higher. ${ }^{7}$ Conserving antibiotic sensitivity through the management of self-limiting respiratory tract infections (RTIs) without recourse to antibiotics is a priority. ${ }^{8}$

Lack of appropriate information about etiological agents results in incongruous practice of drugs. Therefore, the detection of a potentially causative agent forms the precondition for proper treatment and an early detection of virus can moderately decrease the rates of irrational and overuse prescription which leads to the occurrence of an cumulative amount of multi-drug resistant bacteria and infections happens due to bacterial origin have become a global concern. ${ }^{9}$ Hence, the serious illness causing pathogens needs an appropriate regimen to eliminate the situation of worsening antibacterial resistance. The success of an antimicrobial therapy is dependent on a complex relationship between the host, the drug and the invading micro-organism. ${ }^{10}$

Antibiotics are also responsible for the largest number of medicationrelated adverse events, implicated in 1 of every 5 visits to emergency departments for adverse drug reactions. ${ }^{11}$ Adverse events range in severity from mild (for example, diarrhoea and rash) to severe(for example, Stevens-Johnson syndrome, anaphylaxis, Clostridium difficile diarrhea or sudden cardiac death). ${ }^{12}$

Drug utilization research holds a crucial place in clinical practice as it forms the basis for making amendments in the drug dispensing policies at local and national levels. The ultimate goal of such research is to facilitate rational drug use. Also, since it helps in developing strategies to utilize health resources in the most efficient manner, it is particularly needed in a developing economy like India where $72 \%$ of all health care burden is borne by the patients. ${ }^{13}$

Irrational use of antibiotics can increase the risk of adverse effects. Hence, we explore the prescribing pattern of antibiotics in the management of respiratory tract infection and assessment of the drug interactions associated with antibiotics used in respiratory tract infections. 


\section{MATERIALS AND METHODS}

A prospective observational study was conducted on 111 patients for 6 months from October 2019 to April 2020 to evaluate drug utilization pattern of antibiotics. It was observed that different classes of antibiotics (nebulizer, oral and intravenous) were prescribed in Medicine and Respiratory departments. The protocol of the study was approved by Institutional Ethics Committee (IEC) at Maharishi Markandeshwar Institute of Medical Sciences and Research (MMIMSR), Mullana having ethical clearance number: 1554.

A data entry format was specially designed which was used to enter patient demographic data (name, age, gender, address), date of admission, date of discharge, patient social history, medication history, past medical history, diagnosis, type of antibiotic prescribed, dose, side effects etc.

Finally, the results were analyzed using descriptive statistical methods and reported to concerned departments.

Study site: The study was carried out in various departments (General Medicine and Respiratory) of Maharishi Markandeshwar Institute of Medical Sciences and Research (MMIMSR), Mullana, Ambala, Haryana.

Inclusion criteria: Inpatients and Out patients above 18 years who diagnosed with respiratory tract infection and patients willing to participate are included in the study.

Exclusion criteria: Patients below 18 years of age, pregnant and lactation women, psychiatric patients, patient under critical condition and requiring critical care stay, patients having Tuberculosis and Diabetes Mellitus, patients suffering with other than respiratory tract infections, patients not willing to sign inform consent form were excluded from the study.

\section{RESULTS}

All subjects satisfy the inclusion and exclusion criteria were included as the study population. Total 111 inpatient and outpatients' subjects prescribed antibiotics were included in the study in which 75 (67.6\%) were male and $36(32.4 \%)$ were female. Out of 111 patients, there were 46 $(40.5 \%)$ cases of non-smokers and 67(59.5\%) were smokers, 41(36.9\%) patients were having alcohol consumption and 70 (63.1\%) were found to be non-alcoholic patients. Study populations were analyzed to distribute them according to area-wise and it was found that $68.5 \%$ patients were belonging to rural area and $31.5 \%$ patients were from urban area as discussed in Table 1.

Subjects were categorized according to disease and out of 111 subjects 49 (44.1\%) were of COPD, 12 (10.8\%) were of B. Asthma, $16(14.4 \%)$ were of Pneumonia, 13(11.7\%) were of Unspecified infection, 9 (8.1\%) were of Otitis media, 1 (1.3\%) were of Supraglottic, 1 (0.9\%) were of Adenotonsillitis and $12(10.8 \%)$ were of Sinusitis. According to age group distribution 7 subjects were in age group of 18-30 years, 22 subjects were in age group of 31-40 years, 32 subjects were in age group of 41-50years, 33 subjects were in age group of 51-60 years, 11 subjects were in age group of 61-70 years, 6 subjects were in age group more than 71 years. The mean age of population was $49(12.05)$ (Mean \pm SD) and the range of age was between 41-50 years old as discussed in Table 2. In COPD patients with Mono/Dual/Poly therapy were10, 30 and 10, B. Asthma 0,10 and 2, Unspecified infection 4, 7 and 2, Otitis media 3, 6 and 0, Supraglottic 1, 0 and 0, Adenotonsillitis 0, 0 and 1and in Sinusitis 1, 9 and 0 as discussed in Table 3. The analysis of prescriptions were done and found that $26.1 \%$ of the prescriptions were without drug interactions and $73.9 \%$ were with interactions as discussed in Table 4 .

The study reveals that most commonly prescribed antibiotics in case of COPD, Asthma, Pneumonia, Unspecified Infection, Otitis media, Adenotonsillitis and sinusitis was found to be Monocef SB $(71.4 \%, 100 \%, 68.8 \%, 53.8 \%, 88.9 \%, 1005,100 \%, 10 \%)$ followed by
Azithromycin $(85.7 \%, 100 \%, 81.3 \%, 61.5 \%, 0 \%, 0 \%, 0 \%, 0 \%)$, Augmentin $(30 \%, 0 \%, 18.8 \%, 15 \%, 4 \%)$, Tazar $(4.1 \%, 16.7 \%, 31.3 \%, 7.7 \%, 11.1 \%)$, Ofloxacin and Supime were only prescribed(80\%), (70\%) in sinusitis respectively. In the study due to antibiotics side effects were found to be COPD $12(24.48 \%)$, B. Asthma 2 (16.66\%), Pneumonia 5(31.25\%), Unspecified infection 2(15.38\%), supraglottic, adenotonsillitis and otitis media were 0 side effects and sinusitis $1(10 \%)$. Stomach-ache, loose stools, itching and rashes were commonly occurring side effects seen in the patients who were prescribed with Monocef SB, Azithromycin and Tazar antibiotics. The analysis of prescriptions were done and found that $26.1 \%$ of the prescriptions were without drug interactions and $73.9 \%$ were with interactions. The majority of interactions were minor $(69.7 \%)$ the followed by Moderate that alter the elimination and pharmacodynamic (16.8\%), severe that prolong the duration of QTC interval (9\%) and Contraindication interactions (4.5\%) were arises due to taking both Ceftriaxone and calcium carbonate together which causes risk of particulate precipitation in lungs, kidneys as discussed in Table 5.

\section{DISCUSSION}

Prescribing patterns of antibiotics for respiratory tract infections vary widely among general practices. There is currently no national clinical guideline relating to antibiotic prescribing for RTIs. Hence, management of RTIs has been a challenge to the physician, mostly due to emergence of multi drug resistance. ${ }^{14}$ The emerging problem of antibiotic resistance has become a major threat to the medical field. Unnecessary and

\begin{tabular}{cc}
$\begin{array}{l}\text { Table 1: Socio-demographic and clinical characteristics of Respiratory } \\
\text { tract infection patients. }\end{array}$ \\
\hline Variables & No. of patients (\%) \\
\hline Gender & $75(67.6 \%)$ \\
Male & $36(32.4 \%)$ \\
Female & \\
Age (Years) & $7(6.3 \%)$ \\
$18-30$ & $22(19.8 \%)$ \\
$31-40$ & $32(28.8 \%)$ \\
$41-50$ & $33(29.7 \%)$ \\
$51-60$ & $11(9.9 \%)$ \\
$61-70$ & $6(5.4 \%)$ \\
$>70$ & \\
Smoking & $67(59.5 \%)$ \\
Smoker & $46(40.5 \%)$ \\
Non- smoker & \\
Alcohol & $41(36.9 \%)$ \\
Alcoholic & $70(63.1 \%)$ \\
Non- alcoholic & $35(31.5 \%)$ \\
Area & $76(68.5 \%)$ \\
Urban & \\
Rural & \\
\hline
\end{tabular}

Note: In this table the study shows that out of 111 subjects $67.6 \%$ of the patients were male and $32.4 \%$ of the patients were female. The highest no. of patients were in the age group lying under $51-60$ years were $29.7 \%$. The addiction history of the patient reveals that $59.5 \%$ were smokers and $40.5 \%$ were non-smokers on the other hand $36.9 \%$ were alcoholic and remaining were non-alcoholic. $31.5 \%$ were belongs to the urban area and $68.5 \%$ were belongs to rural area. 
inappropriately utilization of antibiotics has been a major concern to this ever-growing problem. ${ }^{15}$

The data was analysed using descriptive statistical methods and were reported to concerned departments. All subjects satisfy the inclusion and exclusion criteria were included as the study population. 111 subjects enrolled in the study who were prescribed with antibiotics and admitted to various department of Medicine and Respiratory in Maharishi Markandeshwar Institute of Medical Science and Research (MMIMSR), Mullana, Ambala, Haryana.

Age distribution result shows that there were more number of male patients than female in the study population which was similar to the previous article by Ramakrishna Prudhivi et al. ${ }^{16}$ and maximum population belongs to the age group of 51-60 years. This was also previously done by S Suwitha et al. as discussed in Table $1 .^{17}$

Patients were categorized according to their disease and out of 111 subjects, $21(18.9 \%)$ cases were diagnosed with URTI in which 09(42.85\%) patients had Otitis media, 01(4.76\%) had Supraglottic, $10(47.61 \%)$ had common cold and $01(4.76 \%)$ had Adeno-tonsillitis. In study population $90(81.1 \%)$ cases were diagnosed with LRTI in which 49(54.44\%) were COPD, 12(13.33\%) were B. Asthma, 16(17.77\%) were Pneumonia, 13(14.44\%) were Unspecified infection diagnosed with both URTI and LRTI. In Similar study done by Vinutna Gogineni et al. also shown that maximum number of patients were diagnosed as in COPD and pneumonia. ${ }^{18}$

Patients were also distributed according to co-morbidities so; majority of patients had hypertension $13(11.71 \%)$ as co-morbidity followed by acute exacerbation 12 (10.81\%). Highest number of co-morbidities was seen in single patient i.e. COPD with DM with acute exacerbation as discussed in Table 2.

Patients were analyzed as mono/dual/triple therapy. ${ }^{19}$ In COPD 10, 30 and 10 patients having Mono/Dual/Poly therapy whereas in case of $\mathrm{B}$. Asthma 0, 10, 2 patients in Unspecified infection 4, 7, 2 patients in Otitis

Table 2: Distribution of the study population according to Disease and co-morbidities.

\begin{tabular}{|c|c|c|c|c|c|}
\hline 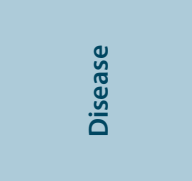 & 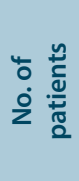 & 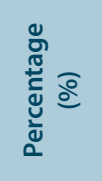 & 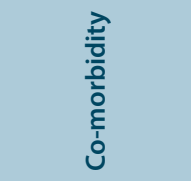 & 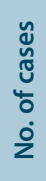 & 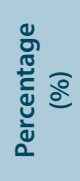 \\
\hline URTI & 21 & $18.9 \%$ & Hypertension & 13 & 11.71 \\
\hline Otitis media & 09 & $42.85 \%$ & $\begin{array}{c}\text { Chronic kidney } \\
\text { disease }\end{array}$ & 01 & 0.90 \\
\hline Supraglottic & 01 & $4.76 \%$ & $\begin{array}{c}\text { Acute } \\
\text { exacerbation }\end{array}$ & 12 & 10.81 \\
\hline Sinusitis & 10 & $47.61 \%$ & $\begin{array}{l}\text { Diabetes } \\
\text { mellitus }\end{array}$ & 06 & 5.40 \\
\hline Adenotonsillitis & 01 & $4.76 \%$ & Pneumothorax & 03 & 2.70 \\
\hline LRTI & 90 & $81.1 \%$ & $\begin{array}{l}\text { Bronchogenic } \\
\text { carcinoma }\end{array}$ & 02 & 1.80 \\
\hline COPD & 49 & $54.44 \%$ & Pleural effusion & 03 & 2.70 \\
\hline B. Asthma & 12 & $13.33 \%$ & Dengue & 02 & 1.80 \\
\hline Pneumonia & 16 & $17.77 \%$ & Gastritis & 02 & 1.80 \\
\hline $\begin{array}{l}\text { Unspecified } \\
\text { infection }\end{array}$ & 13 & $14.44 \%$ & & & \\
\hline
\end{tabular}

*The study revealed the major associated or co-morbid illness categories were Hypertension 13(11.71\%), CKD 01(0.90\%), Acute exacerbation1 2(10.81\%), Diabetes mellitus 06(5.40\%), Pneumothorax 03(2.70\%), Pleural effusion 03(2.70\%), Bronchogenic carcinoma, Dengue and Gastritis were $02(1.80 \%)$. media 3, 6, 0 patients in Supraglottic 1, 0, 0 patients in Adeno-tonsillitis $0,0,1$ patients and in Sinusitis 1, 9, 0 patients were reported, as discussed in Table 3.

In this study $41.4 \%$ patients were undergone culture sensitivity test which helps in providing accurate antibiotic therapy as mentioned in Table 4. Other diagnostic test like X-ray was also performed for all patients. ${ }^{20}$ The decrease in the percentage of culture might be based on the clinical presentation at the time of admission or patient might have consumed the antibiotic prior to admission.

Out of 111 prescriptions, total of 215 Antibiotics were prescribed among which most commonly used is (Monocef SB) Ceftriaxone + sulbactam 76(68.5\%) followed by Azithromycin 72(64.9\%) (Table 5). The data was compared to the study done by Divya Kancherla et al. which concludes that commonly prescribed antibiotic in both URTI and LRTI were ceftriaxone and azithromycin. ${ }^{21}$

The study revealed that 89 patients $(80.10 \%)$ of the prescriptions were found with drug interactions among which $8(9 \%)$ were severe, 15(16.8\%) were moderate, 62(69.7\%) were minor drug interactions and Contraindication interactions were (16.8\%), (9\%), (4.5\%) respectively as discussed in Table 5. Similar study was reported and concluded that $8 \%$ of the reactions were severe and others were moderate and mild according to the Hartwig scale i.e. $68 \%$ and $28 \%$ respectively. ${ }^{22}$ Jimmy Jose et al. also found that moderate and mild reactions were more, but some severe reactions were also found. ${ }^{23}$

The analysis of prescriptions were done and found that $26.1 \%$ of the prescriptions were without drug interactions and $73.9 \%$ were with interactions mentioned in Table 5 . In study conducted by Kancherla Divia et al. revealed that $67.5 \%$ prescriptions had at least 1 interaction and remaining $32.5 \%$ did not have any drug interactions. ${ }^{20}$

\section{CONCLUSION}

Prescription pattern studies have become a potential tool for evaluating the health care systems. The study shows that misuse of antimicrobials may lead to rise in adverse effects, resistance to antimicrobials, making illnesses more severe, and increasing expenditure of health services. The

Table 3: Patient with Mono/Dual/Poly therapy.

\begin{tabular}{cccc}
\hline Disease & Mono-therapy & Dual- therapy & Poly-therapy \\
\hline COPD & 10 & 30 & 10 \\
B. Asthma & 0 & 10 & 02 \\
Pneumonia & 01 & 11 & 04 \\
Unspecified infection & 04 & 07 & 02 \\
Otitis media & 03 & 06 & 0 \\
Supraglottic & 01 & 0 & 0 \\
Adenotonsillitis & 0 & 0 & 1 \\
Sinusitis & 01 & 09 & 0 \\
\hline
\end{tabular}

*The study revealed that in COPD patients with Mono/Dual/Poly therapy were 10,30 and 10 , B. Asthma 0,10 and 2, Unspecified infection 4,7 and 2, Otitis media 3,6 and 0, Supraglottic 1,0 and 0, Adenotonsillitis 0, 0 and 1and in Sinusitis 1, 9 and 0 .

Table 4: Percentage of culture sensitivity-resistance performed.

\begin{tabular}{ccc}
\hline Prescription screened & $\begin{array}{c}\text { No. of } \\
\text { prescription }\end{array}$ & Percentage (\%) \\
\hline Prescription with culture performed & 46 & $41.4 \%$ \\
Prescription without culture performed & 65 & $58.6 \%$ \\
\hline
\end{tabular}

*The study revealed that $58.6 \%$ of the prescriptions were found to be without culture sensitivity performed and $41.4 \%$ were with culture sensitivity performed. 
Table 5: Commonly prescribed antibiotics, side effects, Drug-drug interactions and severity of interactions.

\begin{tabular}{|c|c|c|}
\hline \multicolumn{3}{|c|}{ Commonly Prescribed Antibiotics } \\
\hline Antibiotics & No. of patients & Percentage (\%) \\
\hline Ceftriaxone+Sulbactam & 76 & $68.5 \%$ \\
\hline Azithromycin & 72 & $64.9 \%$ \\
\hline Augmentin & 19 & $17.1 \%$ \\
\hline Piperacillin+Tazobactam & 12 & $10.8 \%$ \\
\hline Ofloxacin & 10 & $9 \%$ \\
\hline Cefepime+sulbactam & 09 & $8.1 \%$ \\
\hline Cefuroxime+Clavulanic acid & 05 & $4.5 \%$ \\
\hline Amoxicillin+Clavulanic acid & 04 & $3.6 \%$ \\
\hline Doxycycline & 03 & $2.7 \%$ \\
\hline Clindamycin & 02 & $1.8 \%$ \\
\hline Clarithromycin & 02 & $1.8 \%$ \\
\hline Tobramycin & 01 & $0.9 \%$ \\
\hline \multicolumn{3}{|c|}{ Side Effects } \\
\hline Condition & No. of patients & Percentage (\%) \\
\hline COPD & 12 & $24.48 \%$ \\
\hline B. Asthma & 2 & $16.66 \%$ \\
\hline Pneumonia & 5 & $31.25 \%$ \\
\hline Unspecified infection & 2 & $15.38 \%$ \\
\hline Otitis media & 0 & 0 \\
\hline Supraglottic & 0 & 0 \\
\hline Adenotonsillitis & 0 & 0 \\
\hline Sinusitis & 1 & $10 \%$ \\
\hline \multicolumn{3}{|c|}{ Drug-drug Interactions } \\
\hline Prescription screened & $\begin{array}{c}\text { No. of } \\
\text { prescription }\end{array}$ & Percentage (\%) \\
\hline Prescription with drug interaction & 82 & $73.9 \%$ \\
\hline $\begin{array}{l}\text { Prescription without drug } \\
\text { interaction }\end{array}$ & 29 & $26.1 \%$ \\
\hline \multicolumn{3}{|c|}{ Severity of Interactions } \\
\hline Severity & $\begin{array}{c}\text { Number of } \\
\text { interactions }\end{array}$ & Percentage (\%) \\
\hline Minor & 62 & $69.7 \%$ \\
\hline Moderate & 15 & $16.8 \%$ \\
\hline Severe & 8 & $9 \%$ \\
\hline Contraindication & 4 & $4.5 \%$ \\
\hline
\end{tabular}

* The study revealed that most commonly prescribed antibiotics were Ceftriaxone+Sulbactam and Azithromycin.

most commonly prescribed antibiotics for respiratory tract infections were Ceftriaxone + sulbactam and azithromycin. Two drug combinations of antibiotics were more prescribed than monotherapy and poly-therapy. From this study, it is concluded that cautious and judicious use of antibiotics will reduce the burden of multi-drug resistance and thereby enabling better patient management and limiting the resultant morbidity and mortality arising from respiratory tract infections. The incidence of medication error was found to be low and there were no life-threatening events.
There is hence a need for educational programmes in order to facilitate rational use of antibiotics and promote sensible use of available resources. Every hospital should also follow standard treatment guidelines taking into consideration the sensitivity pattern of the organisms in the locality to promote rational prescribing.

A clinical pharmacist can also play a major role in early detection and prevention of medication errors and thus can improve the quality of life of the patients by involvement of clinical pharmacist in prescription analysis and prescribing pattern. Studies can help provide feedback to the physicians on the current prescribing practices.

\section{ACKNOWLEDGEMENT}

The authors would like to thank all physicians and nursing staff who participated in this study.

\section{CONFLICT OF INTEREST}

The authors declare that there is no conflict of interest.

\section{ABBREVIATIONS}

RTIs: Respiratory tract infections; URTI: Upper respiratory tract infection; LRTI: Lower respiratory tract infection; COPD: Chronic obstructive pulmonary disease; DM: Diabetes mellitus; AMR: Anti-microbial resistance; IEC: Institutional Ethics Committee; DUE: Drug utilization evaluation.

\section{REFERENCES}

1. World Health Organization. Global health observatory data repository causes of death; 2015.

2. Malladi P, Hasna AH, Ramesh S, Manna PK. Role of clinical pharmacist in promoting rational use of antimicrobial in the management of paediatric lowe respiratory tract infection in a tertiary teaching hospital. Int J Res Pharm Chem. 2012;2(2):360-70.

3. Díez-Domingo J, Pérez-Yarza EG, Melero JA, Sánchez-Luna M, Aguilar MD Blasco AJ, Alfaro N, Lázaro P Social, economic, and health impact of the respiratory syncytial virus: a systematic search. BMC Infect Dis. 2014;14:544 doi: 10.1186/s12879-014-0544-x. PMID 25358423.

4. World Health Organization. Antimicrobial resistance: global report on surveillance; 2014

5. Bell BG, Schellevis F, Stobberingh E, Goossens H, Pringle M. A systematic review and meta-analysis of the effects of antibiotic consumption on antibiotic resistance. BMC Infect Dis. 2014;14:13. doi: 10.1186/1471-2334-14-13, PMID 24405683.

6. Costelloe C, Metcalfe C, Lovering A, Mant D, Hay AD. Effect of antibiotic prescribing in primary care on antimicrobial resistance in individual patients: systematic review and meta-analysis. BMJ. 2010;340:c2096. doi: 10.1136/bmj. c2096, PMID 20483949.

7. National Institute of Clinical Excellence. Clinical knowledge summaries; 2012.

8. World Health Organization. Antimicrobial resistance: global report on surveillance. Vol. 194; 2014

9. Ahmed MM, ELMaraghy AA, Andrawas EW. Study of prescription patterns of antibiotics in treating lower respiratory tract infections at Sohag Chest Hospital. Egypt J Chest Dis Tuberc. 2016;65(1):143-55. doi: 10.1016/j.ejcdt.2015.04.012.

10. Gonzales R, Malone DC, Maselli JH, Sande MA. Excessive antibiotic use for acute respiratory infections in the United States. Clin Infect Dis. 2001;33(6):757-62 doi: 10.1086/322627, PMID 11512079

11. Shehab N, Patel PR, Srinivasan A, Budnitz DS. Emergency department visits for antibiotic-associated adverse events. Clin Infect Dis. 2008;47(6):735-43. doi: 10.1086/591126, PMID 18694344

12. Lessa FC, MuY, Bamberg WM, Beldavs ZG, Dumyati GK, Dunn JR, Farley MM, Holzbauer SM, Meek JI, Phipps EC, Wilson LE, Winston LG, Cohen JA Limbago BM, Fridkin SK, Gerding DN, McDonald LC. Burden of Clostridium difficile infection in the United States. N Engl J Med. 2015;372(9):825-34. doi: 10.1056/NEJMoa1408913, PMID 25714160

13. Mittal N, Mittal R, Singh I, Shafiq N, Malhotra S. Malhotra S. Drug utilisation study in a tertiary Care Center: recommendations for improving hospital drug dispensing policies. Indian J Pharm Sci. 2014;76(4):308-14. PMID 25284928.

14. Ramana KV, Kalaskar K, Rao M, Rao SD. Aetiology and antimicrobial susceptibility pattern of LRTI in a rural tertiary care teaching hospital in Karimnagar, South India. Am J Infect Dis. 2013;1(5):101-05. 
15. Nandimath MK, Ahuja S. Drug prescribing pattern in upper respiratory tract infection in children aged 1-14 years. Int J Bus Soc. 2012;3(1):299-08.

16. NahidAkbari P. Ramakrishna, Naidu Shobha. Rational use of antibiotics and monitoring of drug interactions in patients with respiratory tract infection. J. Pharm. Pract. Commun Med. 2018;4(4):211-15.

17. Suwitha S, Suriya P, Senthilvelan $M$, Baburaj $K$, raja E. A; A study on prescribing pattern of antibiotics in lower respiratory tract infection among adults in tertiary care teaching hospital. J Pharm Innov. 2019;8(7):169-74.

18. VinutnaGogineni BM, NamburiReshma KumariR. Drug utilization study in patients with acute respiratory tract infections at a tertiary care hospital in Bhimavaram, India, Int. J Adv. Res Dev. 2018;3(9):35-6.

19. Mazzaglia G, Arcoraci V, Greco S, Cucinotta G, Cazzola M, Caputi AP. Prescribing habits of general practitioners in choosing an empirical antibiotic regimen for lower respiratory tract infections in adults in Sicily, Pharmacological Research,
Egypt J Chest Dis Tuberc. 1999;40(1):47-2.

20. Harish GN, Chitra C, Ashwini K, Rohit D, Sunil G, et al. Drug utilization study on antibiotics use in lower respiratory tract infection. Indian $\mathrm{J}$ Med Res. 2013;3(4):324-7.

21. Kancherla D, SatyaSai MV, Gayathri Devi HJ, Sharma S. A study on prescribing pattern of antibiotics in respiratory tract infections in a tertiary care center. Int J Recent Sci Res. 2015;6(6):4558-63.

22. Shamna M, Dilip C, Ajmal M, Linu Mohan P, Shinu C, Jafer CP, Mohammed Y. A prospective study on Adverse Drug Reactions of antibiotics in a tertiary care hospital. Saudi Pharm J. 2014;22(4):303-8. doi: 10.1016/j.jsps.2013.06.004 PMID 25161373.

23. Jose, Jimmy, Rao, Padma G.M., Jimmy, Beena, et al. Adverse drug reactions to fluoroquinolone antibiotics analysis of reports received in a tertiary care hospital, Int J Risk Saf Med. 2008; 20:169-80.

Article History: Received 25-04-2021; Revised 17-05-2021;Accepted 03-06-2021

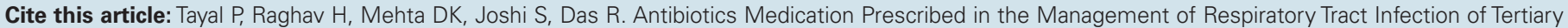
Care Teaching Hospital. J Young Pharm. 2021;13(3):262-6. 